\title{
Optimization of CO2 Emission, Driving Cost and Time Using Pollution Routing Protocol and ST-70 Benchmark
}

\author{
Aakanksha Sharma $^{1}$, Anuradha Panjeta $^{2}$ \\ ${ }^{1,2}$ Department of CSE, Kurukshetra University, Kurukshetra, India \\ 1aakanksha.sharma26@gmail.com²anupanjeta@ rediffmail.com
}

\begin{abstract}
According to Vedic hymn, "The air is the spirit of all deities. It exists in all as life-breath. It is capable to move everywhere. We can't see it. Only we can hear its sound. We are praying to air God." Vehicle is creating and will create $\mathrm{CO} 2$ emission and eventually increase air pollution. Pollution has negative impact on our environment. Therefore, our main objective is to reduce pollution by vehicle. We can't derail development, that's why we can't stop vehicle. Only we can reduce the minimum distance covered by vehicle in an efficient manner to deliver high performance. Therefore, in order to reduce distance covered by vehicle, we are using vehicle routing protocol (VRP) based on genetic algorithm. VRP with consideration of pollution also called pollution routing protocol (PRP). Therefore, this project optimizes $\mathrm{CO} 2$ emission, driving cost and driving time using PRP in order to preserve air and protect air God.
\end{abstract}

Keywords - Greenhouse Emission, Driving Cost, Driving Time, Genetic Algorithm, Vehicle Routing Protocol

\section{Introduction}

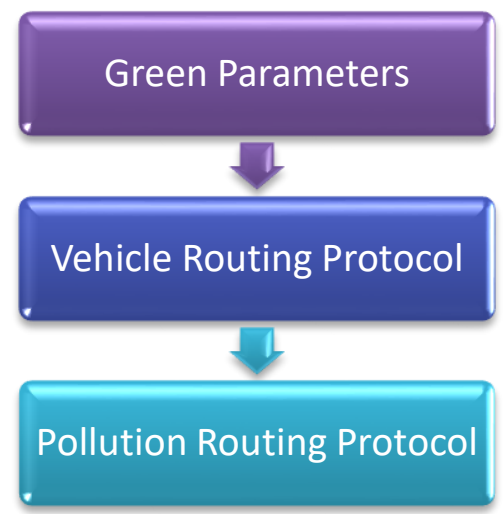

Figure 1: Relation between Pollution Routing Protocol and Vehicle Routing Protocol

This paper deals with Genetic Algorithm \& Vehicle Routing Problem based Pollution Routing Problem (PRP) as shown in Figure 1 with consideration of greenhouse emissions (CO2), fuel, travel distance, travelling time and their costs. 


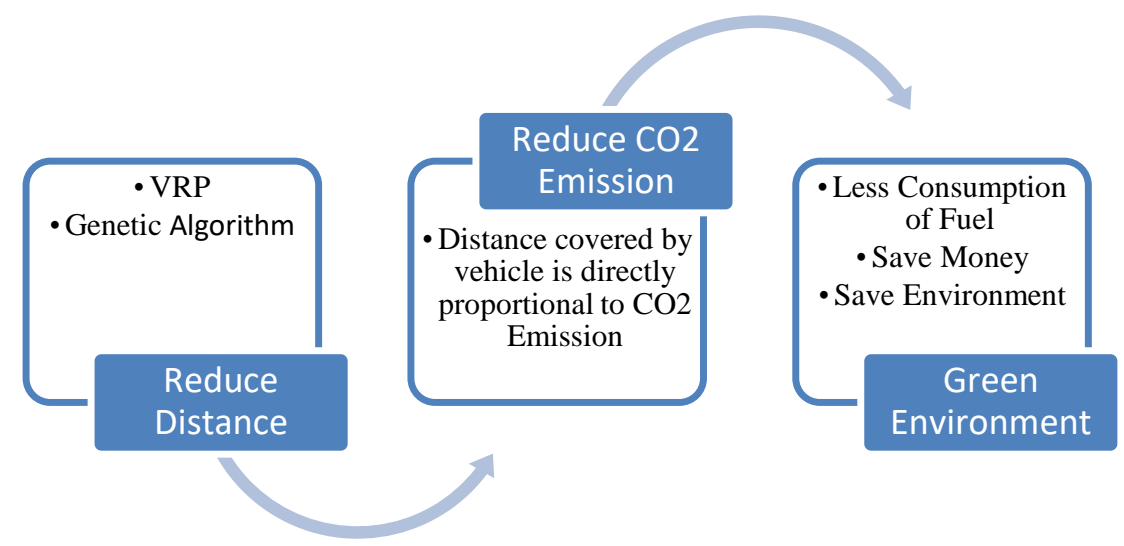

Figure 2: Transformation of VRP into PRP for Green Environment

For problem ST-70 (Result in Table 1-3), there are 70 customers which are going to be served and we assumed that population size of the genetic algorithm is 15 and number of generation is also 15 . We have calculated the distance for that population size and its corresponding fuel consumption, driving time and driver cost are also determined.

\section{Related Work}

The Time-Dependent Pollution-Routing Problem (TDPRP) introduced in [1] and explained with different objectives. Application of genetic algorithm in vehicle routing problem (VRP) with time window constrained is given in [2]. In [3], parallel hybrid genetic algorithm is proposed for VRP with time windows is explained by a masterslave message-passing model. With coordinating genetic operations and deals with selection of parent whereas slave elements simultaneously perform reproduction and mutation operators. For quality optimization the parallel algorithm extends its sequential portion. Multi Depot VRP based on hybrid genetic algorithm is discussed in [4]. A goal programming and genetic algorithm based multi objective vehicle routing problem with time windows (VRPTW) is presented in [5]. The rapid growth of reverse logistics activities make it compulsory evaluate optimized path for simultaneous pick up and deliveries. A genetic algorithm is proposed to solve the problem in [6]. In [8], Vehicular Sensor Network (VSN) is thrust area to monitor city environments in terms of air pollution and Intelligent Transportation Systems. In [8], Choi Okyoung et al. analyze problem in routing data packets with minimum delay in VSN in terms of i) statistics of vehicle traffic ii) cast routing iii) intelligence of future trajectories of vehicles such as buses. Choi Okyoung et al. propose unique road network graph model that incorporates these three factors as routing metric. This paper considers length of the edge. Choi, Okyoung, et al. (8) formulate the packet routing problem as a Markov Decision Process (MDP) based on both network model and delay function, and also develop an optimal routing policy by solving the MDP. Reduction in delay is going to play an important role in reduction of pollution. Reference [9] presents mobility information of vehicles and digital map on city roads based inter-vehicle routing protocol in order to solve those problems in urban area. Mobility information includes the position, velocity, and direction of vehicles. We can calculate distance using available position, velocity, and directions of vehicles. Finally, we can calculate pollution by vehicle in order to cover the distance. In logistic transport industry, individual demands and the diversity requirements are matters in transport operation; [10] focused on the VRP. New genetic algorithm is used to achieve optimized solution in [10]. Initially, we use natural number coding in order to simplify the problem. Secondly, use individual amount control choice strategy to guard the diversity of group; 
approach in [10] also improved route crossover operation to avoid goods damage. Finally, the good performance in terms of minimum distance and minimum pollution of modified algorithm can be proved by experiment calculation and examples [10]. Reference [11] deals with Min Max Vehicle Routing Problem. According to model discussed in this paper, hybrid genetic algorithm is applied for optimized solution [11].

\section{Results}

For problem ST-70 (Result in Table 1-3), there are 70 customers which are going to be served and we assumed that population size of the genetic algorithm is 15 and number of generation is also 15 . We have calculated the distance for that population size and its corresponding fuel consumption, driving time and driver cost are also determined. In Table 1-3, the minimum distance is found to be $694.110 \mathrm{Km}$ and the corresponding fuel consumption, driving time and driving cost is 128.895 Liter, 40.686 hours and $1,220.58$ (INR) respectively. Where remuneration for driver is Rs 30 per $\mathrm{km}$.

A. Analysis of Fuel Consumption to control $\mathrm{CO}_{2}$ Emission

TABLE I. Distance Versus Fuel Consumption in PRP For ST-70

\begin{tabular}{|c|c|}
\hline Distance $(\mathrm{Km})$ & Fuel Consumption (Litre) \\
\hline 709.66 & 131.890 \\
\hline 713.998 & 132.639 \\
\hline 704.099 & 130.800 \\
\hline 697.713 & 129.614 \\
\hline 704.099 & 130.800 \\
\hline 702.582 & 130.518 \\
\hline 754.147 & 140.098 \\
\hline 716.836 & 133.166 \\
\hline 745.960 & 138.577 \\
\hline 718.586 & 133.491 \\
\hline 712.090 & 132.285 \\
\hline 694.110 & 128.895 \\
\hline 695.255 & 129.157 \\
\hline 715.319 & 132.885 \\
\hline 702.582 & 130.518 \\
\hline
\end{tabular}

B. Analysis of Driving Time to deliver High Performance

TABLE II. Distance versus Driving Time in PRP for ST-70

\begin{tabular}{|c|c|}
\hline Distance & Driving Time (Hours) \\
\hline 709.66 & 41.082 \\
\hline 713.998 & 41.183 \\
\hline 704.099 & 40.935 \\
\hline 697.713 & 40.776 \\
\hline 704.099 & 40.935 \\
\hline 702.582 & 40.897 \\
\hline 754.147 & 42.817 \\
\hline 716.836 & 41.254 \\
\hline 745.960 & 41.982 \\
\hline 718.586 & 41.297 \\
\hline 712.090 & 41.135 \\
\hline
\end{tabular}


ISSN: 2456-0065 DOI: 10.21058/gjet.2016.2102

\begin{tabular}{|l|c|}
\hline 694.110 & 40.686 \\
\hline 695.255 & 40.714 \\
\hline 715.319 & 41.216 \\
\hline 702.582 & 40.897 \\
\hline
\end{tabular}

C. Analysis of Driving Cost to Make Cost Effective System

TABLE III. Distance Versus Driving Cost in PRP For ST-70

\begin{tabular}{|c|r|}
\hline Distance $(\mathrm{km})$ & Driver Cost @ 30 (INR) per km \\
\hline 41.082 & 1232.46 \\
\hline 41.183 & 1235.49 \\
\hline 40.935 & 1228.05 \\
\hline 40.776 & 1223.28 \\
\hline 40.935 & 1228.05 \\
\hline 40.897 & 1226.91 \\
\hline 42.817 & 1284.51 \\
\hline 41.254 & 1237.62 \\
\hline 41.982 & 1259.46 \\
\hline 41.297 & 1238.91 \\
\hline 41.135 & 1234.05 \\
\hline 40.686 & 1220.58 \\
\hline 40.714 & 1221.42 \\
\hline 41.216 & 1236.48 \\
\hline 40.897 & 1226.91 \\
\hline &
\end{tabular}

\section{Conclusion}

In this project, we have implemented genetic algorithm for basic vehicle routing problem (assuming there is one vehicle that starts from a depot, serves all the customer and return back to same depot) and determined minimum distance travelled by that vehicle. Then, for that minimum distance, we have calculated fuel consumption, driving time, driver cost and $\mathrm{CO} 2$ emission in order to determine pollution using vehicle routing protocol for different benchmark. Pollution is also reducing with reduction in distance travelled by that vehicle. Eventually, we are contributing for green earth.

\section{Future Scope}

In future, we can extend this project from one vehicle to multiple vehicles and calculate fuel consumption, drive time, driver cost and $\mathrm{CO} 2$ emission for each and every vehicle using optimization principles of pollution routing protocol. There is also an open scope to design pollution aware vehicle in future. Now research communities are moving toward conception of self-driving vehicle. Our approach is an automated distance calculation using genetic algorithm that is eliminating the choice for manual driver that especially need for minimum distance determination using past experience and heuristics. 


\section{References}

1. Franceschetti, A., et.al. The time-dependent pollution-routing problem, Transportation Research Part B: Methodological. 56:265-293, October 2013.

2. Demir, E., Bektaş, T., Laporte, G. The bi-objective Pollution-Routing Problem. European Journal of Operational Research. 232(3):464-478, February 2014.

3. Berger, J., Barkaoui, M., "A parallel hybrid genetic algorithm for the vehicle routing problem with time windows". Computers \& Operations Research. 31(12):2037-2053.

4. Ho, W., George T.S.H., Ji, P., Lau, H.C.W., A hybrid genetic algorithm for the multi-depot vehicle routing problem. Engineering Applications of Artificial Intelligence. 21(4):548-557, June 2008.

5. Ghoseiri, K., Ghannadpour, S.F., "Multi-objective vehicle routing problem with time windows using goal programming and genetic algorithm", Applied Soft Computing. 10(4):1096-1107. Volume 10, Issue 4, September 2010, Pages 1096-1107, ISSN 1568-4946.

6. A. Serdar Tasan, Mitsuo Gen, A genetic algorithm based approach to vehicle routing problem with simultaneous pick-up and deliveries, Computers \& Industrial Engineering, Volume 62, Issue 3, April 2012, Pages 755-761, ISSN 0360-8352.

7. Vahdati, G., Yaghoubi, M., Poostchi, M., \& Naghibi, S. "A New Approach to Solve Traveling Salesman Problem Using Genetic Algorithm Based on Heuristic Crossover and Mutation Operator", International Conference of In Soft Computing and Pattern Recognition (SOCPAR'09), 2009. pp. 112-116.

8. Choi, Okyoung, et al. "Delay-optimal data forwarding in Vehicular Sensor Networks." Modeling \& Optimization in Mobile, Ad Hoc \& Wireless Networks (WiOpt), 2013 11th International Symposium on. IEEE, 2013.

9. Cha, S.H., and Lee . "Mobility Information and Road Topology Based Inter-vehicle Routing Protocol in Urban." Convergence and Hybrid Information Technology. Springer Berlin Heidelberg, 2011. 271-277.

10. Ren, Chunyu, and Shiwei Li. "New Genetic Algorithm for Capacitated Vehicle Routing Problem." Advances in Computer Science and Information Engineering. Springer Berlin Heidelberg, 2012. 695-700.

11. Ren, Chunyu. "Research on Hybrid Genetic Algorithm for Min-Max Vehicle Routing Problem". Advanced Research on Computer Science and Information Engineering. Springer Berlin Heidelberg, 2011. 177-182. 